\section{Hydrocephalus in Wegener's Granulomatosis: Neuroendoscopic Findings and Management}

\section{To the Editor:}

Wegener's granulomatosis (WG) is a systemic disease characterized by inflammatory changes of small and medium-size blood vessels. The clinical features in correlation with histopathology were initially described by Wegener in $1936^{1}$. The disease is associated with granuloma formation and necrosis of the upper airway, lungs, and kidneys ${ }^{2}$, although other organs can also be affected. The nervous system is involved in up to 33\% of cases, with the peripheral nervous system most commonly affected. Involvement of the central nervous system (CNS) is highly unusual ${ }^{3}$. Similarly, hydrocephalus is very uncommon, occurring in only 4 previously documented cases $^{4,5,6,7}$.

We describe an unusual case of WG with pachymeningitis and aqueductal stenosis resulting in hydrocephalus in a patient who underwent neuroendoscopic exploration of the ventricular system. We believe this is the first report of WG-associated hydrocephalus secondary to aqueductal stenosis effectively managed with cerebral endoscopy.

A 33-year-old right-handed white man with WG presented to the emergency department with symptoms of subacute onset of headache, neck and retroorbital pain, diplopia, and blurred vision. He was diagnosed with WG 10 years prior to presentation based on positive antineutrophil cytoplasmic antibodies (ANCA) and nasal sinus biopsy. His condition to date had been stable, with minimal flares consisting of epistaxis, ear infections, skin ulcers in the lower extremities, and a transitory episode of cranial nerve VI palsy. He had been taking immunosuppressants and steroids for years and exhibited morbid obesity with Cushingoid features. Neurological examination revealed mild papilledema and upper gaze palsy, but was otherwise unremarkable.

A noncontrast head computed tomography (CT) scan revealed moderate enlargement of the third and lateral ventricles with mild periventricular edema and thickening of the tentorium (Figure 1A). Magnetic resonance imaging (MRI) with gadolinium demonstrated diffuse thickening and enhancement of the tentorium and pericerebellar meninges (Figure 1B). A sagittal T2-weighted sequence revealed aqueductal stenosis, primarily of the distal third portion (Figure 1C, 1D).

He underwent a neuroendoscopic third ventriculostomy. During the procedure the premammillary and Lilliequist membranes were abnormally thick and firm and required multiple attempts to perforate (Figure 2). After achieving an optimal-size stoma in both the premammillary and Lilliequist membranes we observed the subarachnoid basal cisterns and the basilar artery and confirmed adequate cerebrospinal fluid (CSF) flow through the third ventriculostomy.

The patient's postoperative course was uneventful. All prior neurological symptoms resolved and he was discharged 2 days after the endoscop- ic procedure. The 1-month followup CT scan of the head showed resolution of hydrocephalus (Figure 1E) and he has not required further intervention in 8 months of followup.

WG is considered to be a disease of the ears, nose, throat, paranasal sinuses, upper airway, lungs, and kidney ${ }^{3}$. Nervous system involvement has been reported in $15 \%-33 \%$ of patients with $\mathrm{WG}^{8}$. Common neurological manifestations include peripheral neuropathy (mononeuritis multiplex) and cranial neuropathy, such as external ophthalmoplegia or hearing loss ${ }^{8}$. Other less common neurological manifestations include intracranial hemorrhage, Horner's syndrome, aseptic meningitis, subarachnoid hemorrhage, cerebritis ${ }^{8}$, basilar artery occlusion, sellar and parasellar mass ${ }^{5}$, stroke $^{9}$, primary brain lesions ${ }^{10}$, CNS vasculitis, and hydrocephalus ${ }^{4,5,6,7}$.

WG-associated hydrocephalus has been reported in only 4 patients ${ }^{4,5,6,7}$, in whom the initial presentation of hydrocephalus made the diagnosis of WG difficult. In these 4 cases, as with our patient, a CT and/or MRI of the brain revealed ventricular dilation as well as diffuse smooth pachymeningeal thickening and enhancement involving the falx cerebri and tentorium cerebelli. Sagittal T2-weighted imaging in this case also revealed aqueductal stenosis of the distal third portion (Figure 1C, 1D).

In the previously reported cases, hydrocephalus was attributed to aseptic inflammatory meningitis. However, the mechanism was unclear and few hypotheses were brought into consideration ${ }^{4}$ : in our patient, hydrocephalus was demonstrated to be secondary to aqueductal stenosis.

Endoscopic management of intraventricular pathology and creation of an internal CSF diversionary channel for hydrocephalus have recently become the preferred option at neurosurgical centers with the required experience. Advantages of cerebral endoscopy include avoidance of shunt malfunctions (e.g., infection, deterioration, breakage, and migrations), avoidance of low pressure syndromes and subdural hematomas/hygromas, and elimination of the morbidity associated with multiple shunt revisions. As patients with WG typically require longterm immunosuppression (which makes them more susceptible to infections, particularly in the presence of a foreign body), cerebral endoscopy may help lower the risk of infection as compared to shunt placement.

This is the first reported case of WG-associated hydrocephalus secondary to aqueductal stenosis in which cerebral endoscopy was performed with a good outcome. Cerebral endoscopy provides potential advantages over other current treatments and should be considered when treating hydrocephalus and intraventricular pathology.

LEONARDO RANGEL-CASTILLA, MD; SEAN MICHAEL BARBER, MD; YI JONATHAN ZHANG, MD, Department of Neurosurgery, The Methodist Neurological Institute, 6560 Fannin Street, Suite 944, Houston, Texas 77030, USA.

Address correspondence to Dr. Rangel-Castilla;

E-mail: LRcastilla@tmhs.org

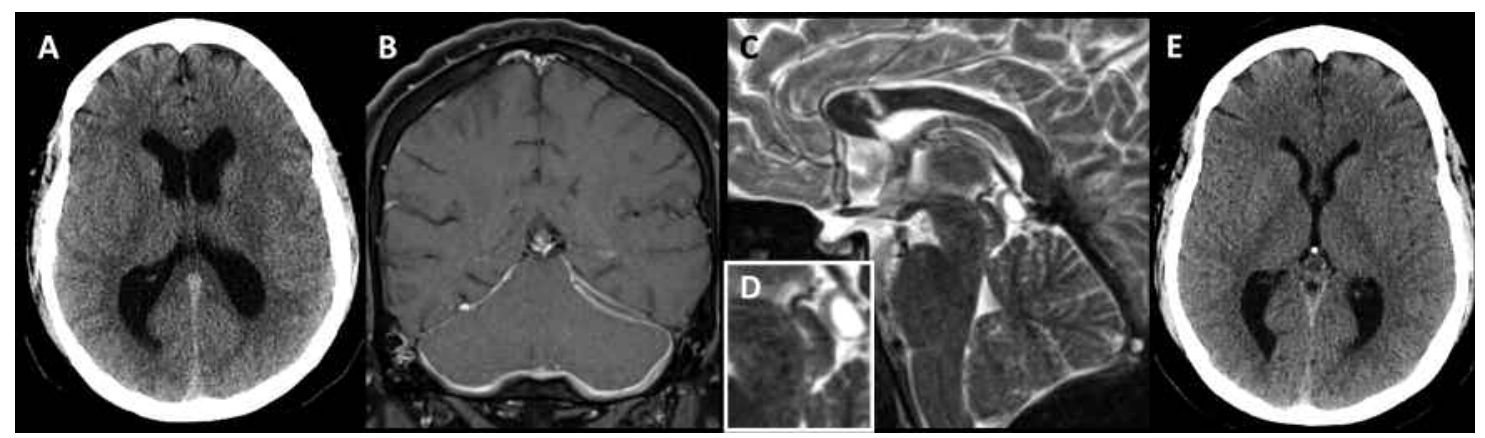

Figure 1. A. Initial noncontrast head CT scan revealing moderate hydrocephalus. B. MRI scan of the brain with gadolinium administration showing pachymeningeal thickening and enhancement of tentorium and infratentorial meninges. C and D. Sagittal T2-weighted midline image showing aqueductal stenosis, primarily of its distal portion. E. At 1-month followup, a head CT scan shows normal-size ventricles. 


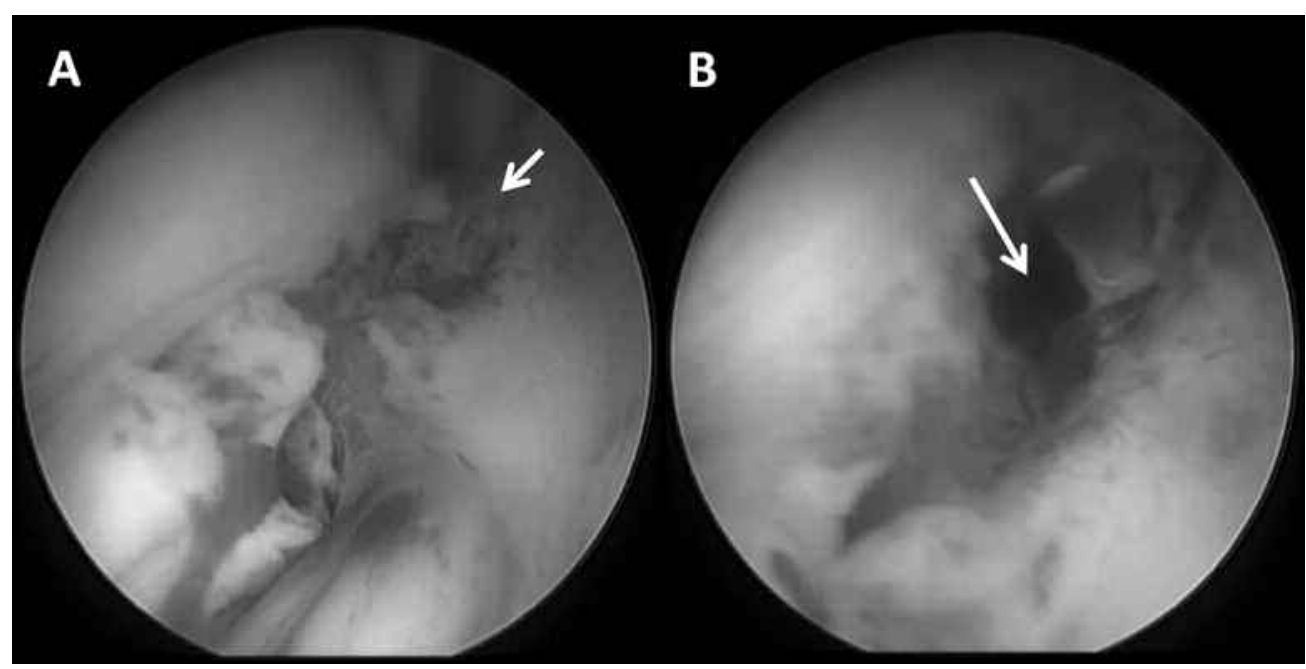

Figure 2. Intraoperative endoscopic images during the third ventriculostomy procedure. A. Floor of the third ventricle after perforation of the tuber cinereum (arrow); note that the Lilliequist membrane had not been pierced at this point. B. After multiple maneuvers, the Lilliequist membrane has been perforated (arrow), creating good communication between the third ventricle and subarachnoid space.

\section{REFERENCES}

1. Wegener F. 50 years of Wegener's granulomatosis. Immun Infekt 1990;18:11-9.

2. Cotch MF, Hoffman GS, Yerg DE, Kaufman GI, Targonski P, Kaslow RA. The epidemiology of Wegener's granulomatosis. Estimates of the five-year period prevalence, annual mortality, and geographic disease distribution from population based data sources. Arthritis Rheum 1996;39:87-92.

3. Jouret F, Noirhomme S, Grandin C, Lonneux M, Godfraind C, Lambert M, et al. Central nervous system involvement in Wegener's granulomatosis. Rev Med Interne 2008;29:912-6.

4. Case records of the Massachusetts General Hospital. Weekly clinicopathological exercises. Case 9-1999. A 74-year-old woman with hydrocephalus and pleocytosis. N Engl J Med 1999;340:945-53.

5. Bertken RD, Cooper VR. Wegener granulomatosis causing sellar mass, hydrocephalus, and global pituitary failure. West J Med 1997;167:44-7.
6. Koga H, Oochi N, Osato S, Ishida I, Hirakata H, Okuda S, et al. Case report: Wegener's granulomatosis accompanied by communicating hydrocephalus. Am J Med Sci 1994;307:278-81.

7. Scarrow AM, Segal R, Medsger TA Jr, Wasko MC. Communicating hydrocephalus secondary to diffuse meningeal spread of Wegener's granulomatosis: case report and literature review. Neurosurgery 1998;43:1470-3.

8. Nishino H, Rubino FA, Parisi JE. The spectrum of neurologic involvement in Wegener's granulomatosis. Neurology 1993;43:1334-7.

9. Satoh J, Miyasaka N, Yamada T, Nishido T, Okuda M, Kuroiwa T, et al. Extensive cerebral infarction due to involvement of both anterior cerebral arteries by Wegener's granulomatosis. Ann Rheum Dis 1988;47:606-11.

10. Miller KS, Miller JM. Wegener's granulomatosis presenting as a primary seizure disorder with brain lesions demonstrated by magnetic resonance imaging. Chest 1993;103:316-8.

J Rheumatol 2011;38:10; doi:10.3899/jrheum.110458 